\title{
Evidence of unexpected oxidative stress in airways of adolescents born very pre-term
}

\author{
Marco Filippone ${ }^{\star,+}$, Gea Bonetto ${ }^{*,+}$, Massimo Corradi ${ }^{\#}$, Anna Chiara Frigo \\ and Eugenio Baraldi*
}

ABSTRACT: Prematurity and its main respiratory complication, bronchopulmonary dysplasia (BPD), are potentially associated with lifelong respiratory morbidities and/or lung function abnormalities. The mechanisms behind these long-term respiratory problems are still unclear.

We assessed airway oxidative stress in adolescents born very pre-term ( $\leqslant \mathbf{3 2}$ gestational weeks) by measuring 8-isoprostane concentration in exhaled breath condensate (EBC). In addition, the study protocol included spirometry and measurement of exhaled nitric oxide fraction (FeNO).

The study groups included 34 ex-pre-term adolescents with BPD, 18 ex-pre-term adolescents without BPD and 34 healthy controls born at term.

Regardless of a history of BPD, the ex-premature adolescents had higher EBC 8-isoprostane levels (median (interquartile range) BPD $9.5(7.3-12.2) \mathrm{pg} \cdot \mathrm{mL}^{-1}$; pre-term non-BPD 10 (8.116) $\left.\mathrm{pg} \cdot \mathrm{mL}^{-1}\right)$ than the controls $\left(3.2(1.9-6.5) \mathrm{pg} \cdot \mathrm{mL}^{-1}\right)(\mathrm{p}<0.001)$. Forced expiratory volume in $1 \mathrm{~s}$ was lower in the BPD group (mean \pm SD Z-score -2.1 \pm 1.58 ) than in the pre-term non-BPD individuals $(-1.13 \pm 1.15)$, who showed in turn significantly lower values than the controls $(0.18 \pm 0.83 ; p<0.001)$. FeNO was similar in the three groups $(p=0.55)$.

Our data show that, after premature birth, evidence of oxidative stress in the airways may be detected into adolescence, suggesting that long-term respiratory abnormalities after pre-term birth may be associated with an ongoing airway disease and not just a stabilised structural lung damage.

KEYWORDS: Bronchopulmonary dysplasia, exhaled breath condensate, 8-isoprostane, prematurity

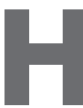

igh rates of premature birth are common in most high-income countries and impose significant health problems and a heavy economic burden on society [1]. Prematurity is associated with a broad spectrum of respiratory symptoms and lung function abnormalities, starting early in life and possibly lasting into adulthood [2]. The most severe forms of pulmonary involvement affect individuals with a diagnosis of bronchopulmonary dysplasia (BPD), the main respiratory complication of prematurity [3, 4]. However, considerable long-term respiratory morbidities have also been reported in formerly premature individuals who developed no BPD [5, 6]. Several studies have documented considerable respiratory symptoms and airflow limitation persisting into young adulthood [6-8] in these subjects, raising concerns that they may be at increased risk of developing a chronic obstructive pulmonary disease (COPD)-like phenotype with ageing $[4,9,10]$. Unfortunately, no pathological data are available to elucidate which structural and pathophysiological changes underlie the clinical and functional pulmonary abnormalities seen in the long term in some prematurely delivered individuals. A relevant question is whether the long-term pulmonary consequences of prematurity and BPD depend essentially on a nonprogressive reduction in airway calibre, due to stabilised early airway remodelling processes and disrupted pulmonary growth, or whether they also reflect an ongoing, active airway disease. The presence of an ongoing airway disease would point to a greater risk of anticipated or accelerated lung function decline with aging in prematurely born individuals, as seen in adults with COPD. However, no established markers of airway disease activity currently exist, despite massive research efforts focusing on asthma and COPD [11].

A potentially relevant indicator of ongoing airway disease is oxidative stress [12], which derives from an increased production of reactive oxygen species and/or decreased antioxidant defences. Markers of oxidative stress are often overexpressed in the airways in several chronic lung disorders, and they are linked to ongoing airway inflammation and remodelling [12]. Oxidative stress has also been consistently associated with lung injury early in life, in infants who progress towards BPD [13], and

\section{AFFILIATIONS}

*Dept of Pediatrics, Unit of Allergy and Respiratory Medicine University of Padua

"Dept of Environmental Medicine and Public Health, University of Padua, Padua, and

\#Dept of Clinical Medicine, Nephrology and Health Sciences, University of Parma, Parma, Italy. +These authors contributed equally to this article.

\section{CORRESPONDENCE}

E. Baraldi

Dept of Pediatrics

Unit of Allergy and Respiratory

Medicine

University of Padua

Via Giustiniani 3

Padua

Italy

E-mail: baraldi@pediatria.unipd.it

Received:

Oct 242011

Accepted after revision:

Feb 152012

First published online:

March 092012 
there is increasing evidence to link early exposure to oxidative stress with potentially lifelong consequences [14]. Oxygen radicals have many complex effects on the body's homeostasis, being involved in normal organ development and contributing to several physiological functions [13]. Recent findings indicate that reactive oxygen species act as second messengers for transcription factor activation and control gene expression [15], and therefore modulate cell growth, apoptosis and inflammation. Any impairment of the oxidative/antioxidative balance may durably harm the newborn, and several studies recommend limiting exposure to oxygen early in life as much as possible [16]. Despite increasing interest in its involvement in lung injury early in life, oxidative stress has yet to be assessed in long-term survivors of premature birth or BPD.

With a view to assessing airway oxidative stress at long-term after pre-term birth, we studied a group of adolescents born prematurely, with and without a history of BPD, by measuring exhaled breath condensate (EBC) concentrations of 8-isoprostane, one of the most reliable biomarkers of oxidative stress in vivo $[17,18]$. EBC affords a noninvasive means for sampling the airways and measuring biomarkers of airway inflammation and oxidative stress $[17,19]$.

\section{METHODS}

\section{Population and study design}

We recruited a group of survivors of BPD and ex-premature subjects with no history of BPD admitted to the Neonatal Intensive Care Unit at the Paediatrics Department, University of Padua, Padua, Italy, between January 1987 and December 1994. We only considered those delivered at a gestational age $\leqslant 32$ weeks for participation in this study.

According to the definition used at the time [20], the BPD group consisted of subjects who had persistent oxygen dependence, clinical signs of respiratory impairment and chest radiograph abnormalities at 28 days of age. To evaluate the possible influences of BPD severity on study results, patients who were oxygen dependent for $\geqslant 28$ days were also classified as cases of mild, moderate or severe BPD according to their need for supplemental oxygen at 36 post-menstrual weeks [3].

The non-BPD premature group included only children who were ventilator-dependent for $<7$ days and oxygen-dependent for $<10$ days after birth. These enrolment criteria were designed so as to evaluate the impact of prematurity itself on long-term respiratory function and $\mathrm{EBC}$ composition, minimising the effect of prolonged mechanical ventilation or otherwise complicated clinical courses.

A group of healthy adolescents born at term with no history of atopic or respiratory diseases was enrolled as a control group.

For lung function assessment and EBC collection, the subjects had to have been in stable clinical conditions, with no upper or lower respiratory tract infections and no need for inhaled or systemic steroids, $\beta_{2}$-agonists or leukotriene receptor antagonists during the previous 4 weeks. All measurements were obtained in the afternoon.

Personal medical history, personal cigarette consumption, and occurrence of wheeze, cough, and exercise-associated symptoms were recorded. Wheeze was considered when diagnosed by a doctor at any time during the previous 2 yrs, as evaluated by the patients' personal medical booklet. Cough was diagnosed if self-reported and occurred on a regular basis either during the day or night in the previous 2 yrs. Exercise-associated symptoms included self-reported cough, wheeze, shortness of breath or chest tightness associated with physical activity, or regular use of inhaled albuterol before exercising.

All subjects underwent a complete physical examination, exhaled nitric oxide fraction ( $F$ eNO) measurement, spirometry, EBC collection and a skin prick test. They were adequately instructed and trained before proceeding with the procedures.

The study was reviewed and approved by the Ethics Committee of the Hospital of Padua, Padua, Italy, and all participants and their parents gave their written informed consent.

\section{EBC collection and 8-isoprostane assay}

EBC was collected and processed according to the European Respiratory Society (ERS)/American Thoracic Society (ATS) recommendations [19]. It was collected using a TURBO-DECCS condenser (Italchill, Parma, Italy), as explained previously [21, 22]. The temperature was kept constant $\left(-4^{\circ} \mathrm{C}\right)$ during the collection. The device is supplied with a disposable respiratory system that consists of a mouthpiece equipped with a one-way valve and a reliable saliva trap, connected to a collecting vial $(50 \mathrm{~mL})$ by means of a tube [22]. The participants breathed tidally through the mouth for $15 \mathrm{~min}$, wearing a nose-clip and sitting comfortably. They kept their mouth dry by periodically swallowing excess saliva. EBC samples were stored at $-80^{\circ} \mathrm{C}$ in polypropylene tubes until assayed, which was performed concurrently for the three groups within 8 months of samples collection. 8-isoprostane was assayed using an enzyme-linked immunoassay (EIA) (Cayman Chemical, Ann Arbor, MI, USA) and its concentrations in EBC were measured by plotting the values identified in the sample with the 8-iso-prostaglandin $(\mathrm{PG}) \mathrm{F}_{2 \alpha}$ standard calibration curve $\left(0-500 \mathrm{pg} \cdot \mathrm{mL}^{-1}\right)$. The lowest detection limit was $3.7 \mathrm{pg} \cdot \mathrm{mL}^{-1}$. For samples below the lowest detection limit, data were arbitrarily expressed as half the detection limit [23]. This assay was previously validated using a reference analytical method (gas chromatographic/negative ion chemical ionisation mass spectrometry) [23]. The repeatability of the EIA 8-isoprostane measurements in EBC on two different days had previously been tested at our laboratory, showing a coefficient of repeatability of $3.2 \mathrm{pg} \cdot \mathrm{mL}^{-1}$. The inter-assay variability was $<15 \%$ [24].

\section{Lung function test}

Lung function was assessed using a 10-L bell spirometer (Biomedin, Padua, Italy) according to international recommendations [25]. Forced vital capacity (FVC), forced expiratory volume in $1 \mathrm{~s}(\mathrm{FEV} 1)$, and forced expiratory flow rate at $25-75 \%$ of FVC (FEF25-75\%) were measured and expressed as Z-scores, calculated using the reference ranges of STANOJEVIC et al. [26].

Spirometry was also performed after administering $400 \mu \mathrm{g}$ of inhaled salbutamol using a metered-dose inhaler with a spacer device (Aerochamber, Trudell, Canada). Reversibility to $\beta_{2^{-}}$ agonists was defined as a $>12 \%$ increase in FEV1 after salbutamol inhalation.

\section{FeNO measurement}

FeNO was measured with the NIOX system (Aerocrine, Stockholm, Sweden), using a single-breath online method 
according to the ATS/ERS guidelines for measuring FeNO in children [27]. In brief, subjects inhaled nitric oxide-free air to total lung capacity and exhaled through a dynamic flow restrictor with a target flow of $50 \mathrm{~mL} \cdot \mathrm{s}^{-1}$ for $10 \mathrm{~s}$.

\section{Skin prick tests}

All subjects underwent skin prick testing with a panel of common inhalant allergens, i.e. mixed grass pollen, Parietaria, Artemisia vulgaris, Dermatophagoides pteronissynus and D. farinae, Alternaria, dog and cat (Lofarma, Milan, Italy). The skin tests were considered positive if they resulted in a wheal reaction $>3 \mathrm{~mm}$.

\section{Statistical analysis}

Results were expressed as mean \pm SD or median (interquartile range (IQR)) depending on the data distribution. The KruskallWallis test, followed by Dunn's test for pairwise comparisons, was used to determine differences between the study groups. Correlations were drawn with Pearson's and Spearman's tests for normally and non-normally distributed data, respectively. Power calculations revealed that the sample size of the study enables detection with the one-way ANOVA an effect size of at least 0.24 in EBC 8-isoprostane with a power of $>90 \%$ at a twosided $\alpha$ level of 0.05 (nQuery Advisor 6.01; Statistical Advisor, Cork, Ireland). The statistical analysis was performed using the software SAS version 9.1.3 for Windows (SAS Institute Inc., Cary, NC, USA).

\section{RESULTS}

34 BPD survivors and 18 non-BPD premature adolescents met the inclusion criteria and agreed to take part in the study. 34 healthy children born at term formed a control group.

The participants' anthropometric characteristics and relevant details of their neonatal history are given in table 1 . None of the participants were cigarette smokers. Among BPD survivors, at 36 postmenstrual weeks, 25 individuals were still on supplemental oxygen (moderate-severe BPD) and nine were breathing room air (mild BPD).

\section{Respiratory symptoms}

$17(50 \%)$ of the 34 survivors of BPD and eight (44\%) of the 18 non-BPD premature participants had recurrent wheezing in the first 5 yrs of life. 10 (29\%) individuals in the BPD group did not refer with any respiratory disturbance in the past 2 yrs. Among the remaining 24 subjects of the BPD group, five referred with occurrence of cough, 13 with exercise-associated symptoms, and 17 with past or current use of inhaled steroids and/or $\beta_{2}$-agonists. 10 (55\%) of the 18 premature non-BPD individuals did not mention any respiratory problem in the past 2 yrs, but six reported exercise-associated symptoms, one cough, one wheeze and two the use of inhaled medications.

\section{EBC 8-isoprostane}

The 8-isoprostane concentrations in EBC were similar in the two pre-term-born groups, with and without a history of BPD (median (IQR) 9.5 (7.3-12.2) and $10(8.1-16) \mathrm{pg} \cdot \mathrm{mL}^{-1}$, respectively), and were significantly higher than in the healthy control group (3.2 (1.9-6.5) $\left.\mathrm{pg} \cdot \mathrm{mL}^{-1} ; \mathrm{p}<0.01\right)$ (fig. 1).

Among the pre-term-born participants, with and without a history of BPD, the 8-isoprostane levels were unrelated to age $(p=0.32)$, height $(p=0.58)$ and weight $(p=0.52)$ at study participation, to gestational age $(r=0.006, p=0.96)$, gestational age-adjusted birthweight Z-score $(\mathrm{r}=0.09, \mathrm{p}=0.58)$, time on mechanical ventilation $(\mathrm{r}=0.036, \mathrm{p}=0.82)$ or oxygen dependence $(r=0.099, p=0.52)$. No correlation was found in these subjects between EBC 8-isoprostane concentrations and spirometric variables (FEV1, $\mathrm{p}=0.075 ; \mathrm{FEV} 1 / \mathrm{FVC}, \mathrm{p}=0.15$; FEF25-75\%, $p=0.065)$, the percentage increase in FEV1 after inhaled albuterol administration $(\mathrm{p}=0.17)$ or FeNO $(\mathrm{p}=0.64)$. EBC 8-isoprostane levels were similar in mild and moderateto-severe survivors of BPD (9.8 (7.6-11.9) and 9.2 (7.113.1) $\mathrm{pg} \cdot \mathrm{mL}^{-1}$, respectively; $\left.\mathrm{p}=0.89\right)$, in atopic and nonatopic participants $(p=0.36)$, and in subjects with and without respiratory symptoms during the past 2 yrs $(p=0.27)$.

\section{Spirometry}

Significant differences emerged between groups by comparison of all spirometric parameters (fig. 2). A marked airflow limitation was found in the BPD group, showing significantly

TABLE 1 Neonatal data and anthropometric characteristics of participants

\begin{tabular}{lccc} 
& BPD & Pre-term non-BPD & Full-term controls \\
\hline Subjects $\mathbf{n}$ & 34 & 18 & 34 \\
Males/females $\mathbf{n}$ & $16 / 18$ & $9 / 9$ & $20 / 14$ \\
Gestational age weeks & $29(27-30)$ & $28(26-30)$ & $39(38-40)$ \\
Birthweight g & $990(847-1157)$ & $940(840-990)$ & $3350(3000-3600)$ \\
Surfactant treated & $20(59)$ & $5(28)$ & $1(1-3.2)$ \\
Mechanical ventilation days & $20.5(11-32)$ & $2(1-5.5)$ & $15.1 \pm 1.7$ \\
O $_{2}$ dependence days & $85(41.3-157.5)$ & $13 \pm 1.9^{* *}$ & $169 \pm 8.3$ \\
Age at study participation yrs & $14.9 \pm 1.9$ & $154 \pm 13.6^{* *}$ & 0.001 \\
Height $\mathbf{~ c m}$ & $160 \pm 11^{* *}$ & $47.1 \pm 12.8^{* *}$ & 0.003 \\
Weight $\mathbf{~ g ~}$ & $50 \pm 10.9^{* *}$ & 0.003 \\
\hline
\end{tabular}

Data are presented as median (interquartile range), $\mathrm{n}(\%)$ or mean $\pm \mathrm{SD}$, unless otherwise stated. BPD: bronchopulmonary dysplasia. ${ }^{* \star}$ : $\mathrm{p}<0.01$ as compared with full-term control group. 


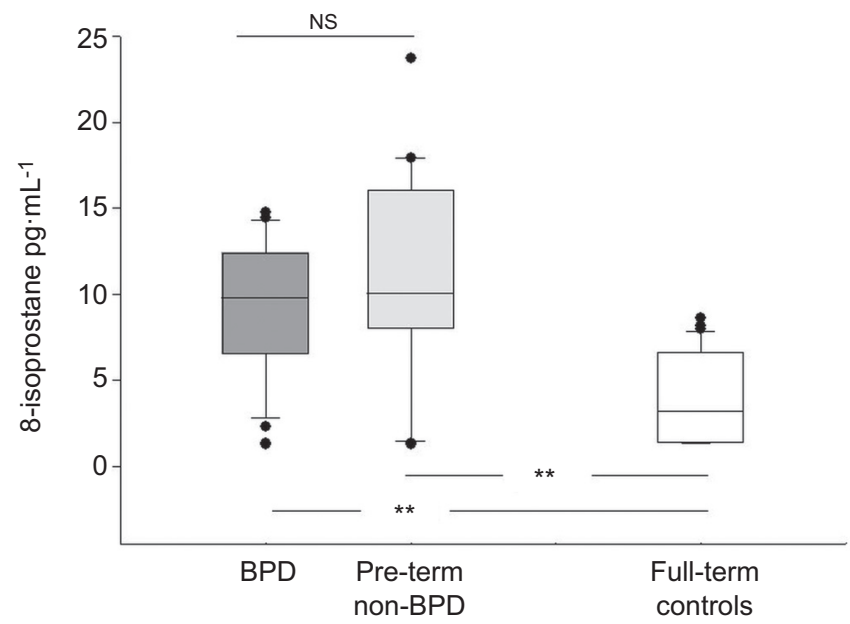

FIGURE 1. Levels of 8-isoprostane in the exhaled breath condensate of adolescents born very pre-term, with and without a history of bronchopulmonary dysplasia (BPD), and of a group of healthy controls born at full term. Boxes represent median values and interquartile ranges; bars represent the 10th and the 90th centiles of the measured concentrations. NS: nonsignificant. ${ }^{* *}: p<0.01$.

lower results for all the spirometric variables $(\mathrm{p}<0.01)$ than for the healthy controls (fig. 2). FEV1 and FEF25-75\% were also significantly reduced in the BPD survivors as compared with the pre-term non-BPD group $(\mathrm{p}<0.05)$. In turn, FEV1 and FEF25-75\% were significantly lower in the pre-term non-BPD adolescents than in the control group (fig. 2).

Airway obstruction was reversible in $35 \%$ of the cases in the BPD survivor group (12 out of 34 ) and in $17 \%$ of the pre-term non-BPD cases (three out of 18).

\section{FeNO}

No differences in FeNO levels emerged between the three groups (BPD 11.4 (8.4-15.7), pre-term non-BPD 14.1 (8.4-17.1) and controls $12.7(7.9-19.2) \mathrm{ppb} ; \mathrm{p}=0.552)$. As atopy is known to affect FeNO levels, a subgroup analysis was performed on the individuals in the three groups with negative skin prick test results, which again revealed no differences in FeNO values.

\section{Skin prick tests}

The skin prick tests for common aeroallergens were positive in six $(18 \%)$ out of 34 in the BPD cases, three $(17 \%)$ out of 18 in the pre-term non-BPD cases and four (12\%) out of 34 in the healthy controls.

\section{DISCUSSION}

This study provides evidence of increased oxidative stress in the airways of adolescents born prematurely, with and without a history of BPD, as reflected by significantly higher EBC 8isoprostane concentrations than in healthy controls born at term. This finding prompts the hypothesis that the long-term chronic respiratory disease following premature birth not only stems from a stabilised structural damage [28], but may also be associated with an ongoing airway disease.

Lung function abnormalities are detectable early in life after premature birth [4, 29], and carry a substantial risk of long-term respiratory disease [30] because a poor lung function in the first months of life is known to track over time with a greater risk of airway obstruction in childhood [31] and young adulthood [32].

The lung function limitations and respiratory symptoms seen in long-term survivors of premature birth and BPD have no clear pathological background to refer to because no biopsy studies have been conducted to elucidate lung morphology in the medium and long term after premature delivery. The airflow obstruction seen in the childhood and adolescence of individuals born pre-term has often been interpreted as the result of stabilised, not progressive structural damage to the airways and/or poor alveolar growth [28].

Data from longitudinal studies on lung function at multiple time-points in adolescents with a history of BPD indicate that lung function may deteriorate over time in some patients $[7,33]$, suggesting that their respiratory disease may be progressive in nature. In addition, the persistence of significantly more frequent respiratory symptoms in adults born even only slightly pre-term $[5,6]$ arouses the clinical suspicion of active airway disease in these patients. Unfortunately, given the lack of morphological information and randomised therapeutic trials, the pathogenesis of such long-term symptoms remains elusive and their treatment empirical.

For this reason it is important to seek indirect information on long-term airway status after premature birth. Analysing EBC may be a useful study tool, being noninvasive, simple to perform and effort-independent. Despite some technical limitations, relating mainly to EBC dilution issues [34], recent literature supports its use as an indirect method for sampling the composition of the airway lining fluid [17, 19, 35]. We focused on 8-isoprostane, a $\mathrm{PGF}_{2 \alpha}$ isomer formed by arachidonic acid peroxidation catalysed by free radicals. The isoprostanes are among the most reliable biomarkers of lipid peroxidation and oxidative stress because of their chemical stability, specificity for lipid peroxidation, in vivo production and relative abundance in biological fluids [17, 18]. 8isoprostane is an important component of the isoprostane pathway and has been found to have powerful biological effects; it may therefore be a mediator and marker of oxygen radical injury [18], a mechanism involved in the pathogenesis of several chronic lung diseases [36], including COPD [12].

The higher concentrations of 8-isoprostane found in the EBC of adolescents born pre-term indicate a three-fold lipid peroxidation rate in their airways by comparison with healthy controls born at term (fig. 1). It is worth noting that no differences emerged between the 8-isoprostane concentrations found in BPD survivors and the adolescents born pre-term with no history of BPD (fig. 1), suggesting that long-term airway oxidative stress is a feature of prematurity per se, regardless of any development of BPD or exposure to prolonged mechanical ventilation and oxygen therapy in neonatal age.

Premature birth in itself poses a considerable oxidative challenge, as pre-term infants abruptly leaving the normally hypoxic intrauterine environment are presented with a sharp increase in oxidative load at a time when they still lack adequate specific defences [13]. Indeed, extremely premature infants show evidence of oxidative stress persisting for several weeks after a brief exposure to high concentrations of inspired oxygen at birth [16]. 
a)
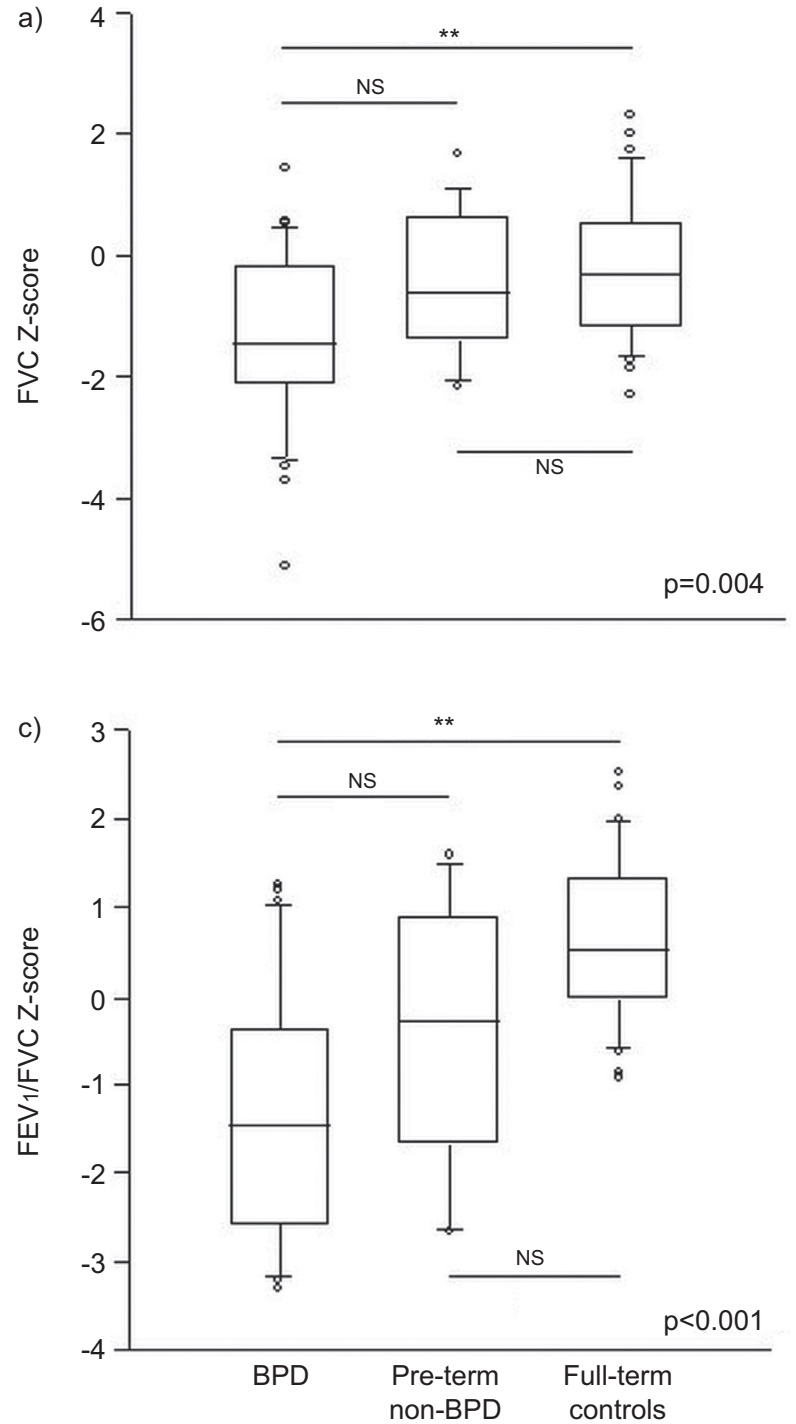

b)

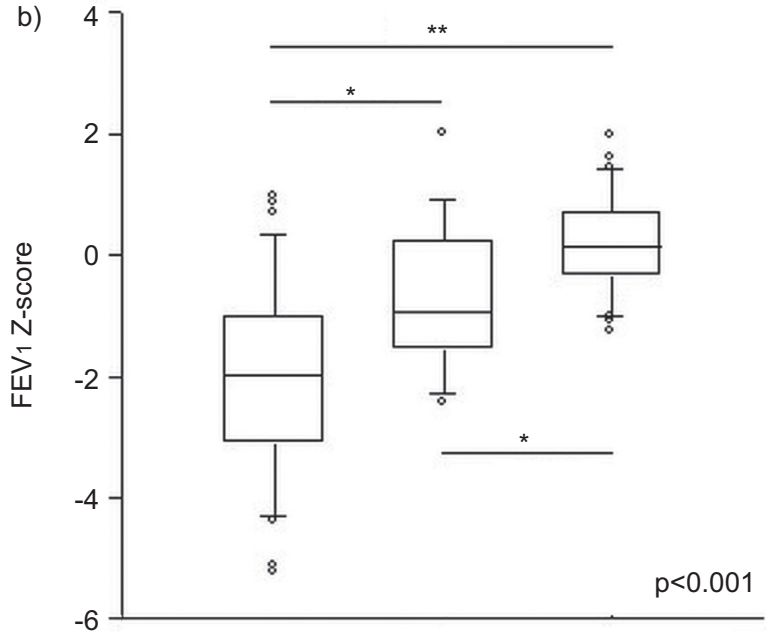

d)

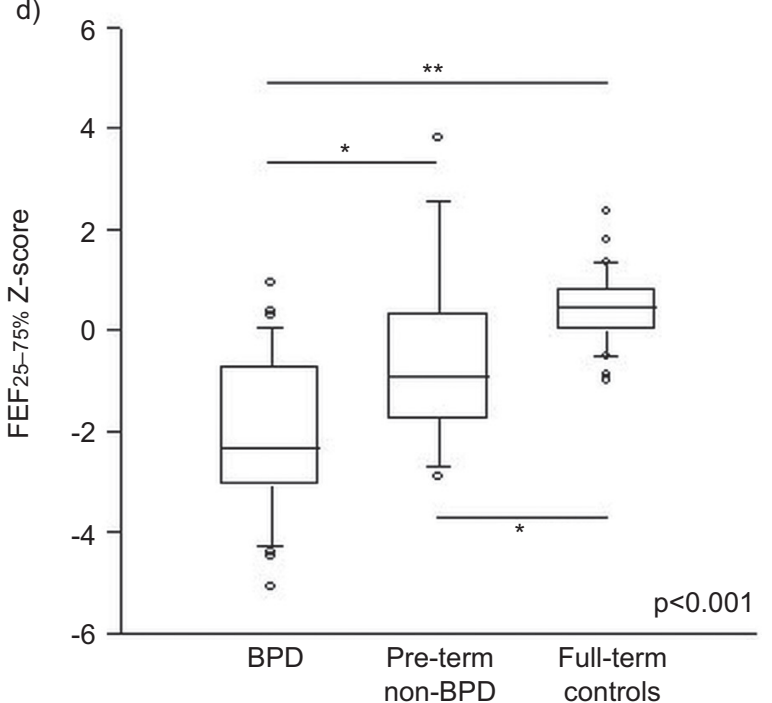

FIGURE 2. Lung function results for a) forced vital capacity (FVC), b) forced expiratory volume in $1 \mathrm{~s}(\mathrm{FEV} 1), \mathrm{c}) \mathrm{FEV} 1 / \mathrm{FVC}$ and d) forced expiratory flow at $25-75 \%$ of $F V C$ (FEF25-75\%) in the bronchopulmonary dysplasia (BPD), pre-term non-BPD and control groups. Data are expressed as Z-scores. Boxes represent median values and interquartile ranges; bars represent the 10th and the 90th centiles of the measured values. NS: nonsignificant. *: $p<0.05 ;{ }^{* *}: p<0.01$.

The 8-isoprostane levels in the EBC of our prematurely delivered participants did not correlate with any of the spirometric variables reflecting airflow, which were significantly worse (as expected) in survivors of BPD. The 8-isoprostane levels in EBC were much the same in the subjects with and without respiratory symptoms in the previous 2 yrs. This suggests that lung underdevelopment and early airway injury have a central role in determining the extent of airway obstruction. We therefore believe that the measurement of oxidative stress provides complementary biological information to lung function in evaluating the pathophysiological processes underlying the long-term pulmonary sequelae of pre-term birth. Whether increased values of 8-isoprostane imply a risk of more severe lung function deterioration later in life remains to be seen.

The FeNO values recorded in the pre-term-born individuals in our series were normal, suggesting that there is no predominant eosinophil-mediated inflammatory pathway in these subjects' airways [37], whereas increased 8-isoprostane levels in preterm-born cases point to active oxidative stress. The association of an increased oxidative stress with normal FeNO values might represent an apparent paradox. Nitric oxide in the lung is a signalling molecule in a wide variety of biological and homeostatic processes including airway and vascular smooth muscle relaxation and neurotransmission. However, there are reports of an increased oxidative stress with normal-to-low values of exhaled nitric oxide in other chronic respiratory diseases, such as cystic fibrosis and COPD [38]. In these diseases a neutrophilic cellular pathway is predominant in the lung and this can explain the paradox of active oxidative stress with normal FeNO levels. Likewise, it could be speculated that a similar neutrophilic inflammation may develop after premature birth and BPD. There are no pathological data to support this hypothesis, however, due to a lack of information on the presence and nature of airway inflammation beyond infancy in subjects born prematurely. 
Whatever its origin and nature, the long-term presence of oxidative stress in the airways after premature birth may be reminiscent of findings consistently reported in the airways of patients with COPD [12]. Oxidative stress plays a crucial part in many of the pathogenic mechanisms behind COPD, and it is associated with protease/antiprotease imbalance and matrix injury, triggering pro-inflammatory activity and alveolar cell apoptosis [12]. Recent hypotheses suggest that cigarette smoking, the principal causative factor of COPD, mainly plays a role as the initial trigger of the disease, which is then maintained through chronic self-supporting injury mechanisms $[39,40]$. We likewise hypothesise that harmful exposures early in life (including oxidative stress) trigger an adaptive disorder in the premature lung that may be maintained, or easily elicited, after the initial injury. The evidence of increased oxidative stress in our prematurely born adolescents might therefore be interpreted as a marker of ongoing airway disease, which may have an impact on these individuals' respiratory health with ageing. The design of the present study prevents any conclusions from being drawn on this issue, as longitudinal data are needed on oxidative stress markers and how they correlate with lung function over time in individuals born prematurely. Further research focusing on other markers of airway oxidative stress, inflammation or remodelling in longterm survivors of prematurity and BPD may help to clarify the natural history of the chronic lung disease that follows premature birth. Additionally, systemic markers of oxidative stress could be targeted for further research in order to evaluate the possible long-term persistence of the "oxygen radical disease" described in the newborn [13].

In conclusion, our finding of a greater oxidative stress in the airways of adolescents born very pre-term suggests the presence of an ongoing airway disease, not just the stabilised structural lung damage assumed until now. This alteration is present regardless of the development of BPD. This issue warrants further investigations into the nature and evolution of the airway disease seen in survivors of prematurity and BPD as they grow older.

\section{STATEMENT OF INTEREST}

A statement of interest for E. Baraldi can be found at www.erj. ersjournals.com/site/misc/statements.xhtml

\section{REFERENCES}

1 Muglia LJ, Katz M. The enigma of spontaneous preterm birth. N Engl J Med 2010; 362: 529-535.

2 Kinsella JP, Greenough A, Abman SH. Bronchopulmonary dysplasia. Lancet 2006; 367: 1421-1431.

3 Jobe AH, Bancalari E. Bronchopulmonary dysplasia. Am J Respir Crit Care Med 2001; 163: 1723-1729.

4 Baraldi E, Filippone M. Chronic lung disease after premature birth. N Engl J Med 2007; 357: 1946-1955.

5 Narang I, Rosenthal M, Cremonesini D, et al. Longitudinal evaluation of airway function 21 years after preterm birth. Am J Respir Crit Care Med 2008; 178: 74-80.

6 Walter EC, Ehlenbach WJ, Hotchkin DL, et al. Low birth weight and respiratory disease in adulthood: a population-based casecontrol study. Am J Respir Crit Care Med 2009; 180: 176-180.

7 Doyle LW, Faber B, Callanan C, et al. Bronchopulmonary dysplasia in very low birth weight subjects and lung function in late adolescence. Pediatrics 2006; 118: 108-113.
8 Halvorsen T, Skadberg BT, Eide GE, et al. Pulmonary outcome in adolescents of extreme preterm birth: a regional cohort study. Acta Paediatr 2004; 93: 1294-1300.

9 Northway WH Jr, Moss RB, Carlisle KB, et al. Late pulmonary sequelae of bronchopulmonary dysplasia. N Engl J Med 1990; 323 1793-1799.

10 Bush A. COPD: a pediatric disease. COPD 2008; 5: 53-67.

11 Vestbo J, Rennard S. Chronic obstructive pulmonary disease biomarker(s) for disease activity needed - urgently. Am J Respir Crit Care Med 2010; 182: 863-864.

12 MacNee W, Tuder RM. New paradigms in the pathogenesis of chronic obstructive pulmonary disease I. Proc Am Thorac Soc 2009; 6: $527-531$.

13 Saugstad OD. Oxygen and oxidative stress in bronchopulmonary dysplasia. J Perinat Med 2010; 38: 571-577.

14 Lee JW, Davis JM. Future applications of antioxidants in premature infants. Curr Opin Pediatr 2011; 23: 161-166.

15 Wright CJ, Dennery PA. Manipulation of gene expression by oxygen: a primer from bedside to bench. Pediatr Res 2009; 66: 3-10.

16 Vento M, Moro M, Escrig R, et al. Preterm resuscitation with low oxygen causes less oxidative stress, inflammation, and chronic lung disease. Pediatrics 2009; 124: e439-e449.

17 Borrill ZL, Roy K. K, Singh D. Exhaled breath condensate biomarkers in COPD. Eur Respir J 2008; 32: 472-486.

18 Montuschi P, Barnes PJ, Ciabattoni G. Measurement of 8isoprostane in exhaled breath condensate. Methods Mol Biol 2010; 594: 73-84.

19 Horváth I, Hunt J, Barnes PJ, et al. Exhaled breath condensate: methodological recommendations and unresolved questions. Eur Respir J 2005; 26: 523-548.

20 Northway WH Jr, Rosan RC, Porter DY. Pulmonary disease following respiratory therapy of hyaline-membrane disease: bronchopulmonary dysplasia. N Engl J Med 1967; 276: 357-368.

21 Goldoni M, Caglieri A, Andreoli R, et al. Influence of condensation temperature on selected exhaled breath parameters. BMC Pulm Med 2005; 5: 10.

22 Caglieri A, Goldoni M, Acampa O, et al. The effect of inhaled chromium on different exhaled breath condensate biomarkers among chrome-plating workers. Environ Health Perspect 2006; 114: 542-546.

23 Carraro S, Cogo PE, Isak I, et al. EIA and GC/MS analysis of 8-isoprostane in EBC of children with problematic asthma. Eur Respir J 2010; 5: 1364-1369.

24 Zanconato S, Carraro S, Corradi M, et al. Leukotrienes and 8isoprostane in exhaled breath condensate of children with stable and unstable asthma. J Allergy Clin Immunol 2004; 113: 257-263.

25 Miller MR, Hankinson J, Brusasco V, et al. Standardisation of spirometry. Eur Respir J 2005; 26: 319-338.

26 Stanojevic S, Wade A, Cole TJ, et al. Spirometry centile charts for young Caucasian children: the Asthma UK Collaborative Initiative. Am J Respir Crit Care Med 2009; 180: 547-552.

27 Baraldi E, de Jongste JC, European Respiratory Society/American Thoracic Society Task Force. Measurement of exhaled nitric oxide in children, 2001. Eur Respir J 2002; 20: 223-237.

28 Lum S, Kirkby J, Welsh L, et al. Nature and severity of lung function abnormalities in extremely preterm children at 11 years of age. Eur Respir J 2011; 37: 1199-1207.

29 Latzin $\mathrm{P}$, Roth S, Thamrin C, et al. Lung volume., breathing pattern and ventilation inhomogeneity in preterm and term infants. PLoS One 2009; 4: e4635.

30 Martinez FD. The origins of asthma and chronic obstructive pulmonary disease in early life. Proc Am Thorac Soc 2009; 6: 272-277.

31 Håland G, Carlsen KC, Sandvik L, et al. Reduced lung function at birth and the risk of asthma at 10 years of age. N Engl J Med 2006; 355: 1682-1689.

32 Stern DA, Morgan WJ, Wright AL, et al. Poor airway function in early infancy and lung function by age 22 years: a non-selective longitudinal cohort study. Lancet 2007; 370: 758-764. 
33 Filippone M, Bonetto G, Cherubin E, et al. Childhood course of lung function in survivors of bronchopulmonary dysplasia. JAMA 2009; 302: 1418-1420.

34 Effros RM. Exhaled breath condensate: delusion or dilution? Chest 2010; 138: 471-472.

35 Montuschi P. Analysis of exhaled breath condensate in respiratory medicine: methodological aspects and potential clinical applications. Ther Adv Respir Dis 2007; 1: 5-23.

36 Morrow JD, Roberts LJ. The isoprostanes: their role as an index of oxidant stress status in human pulmonary disease. Am J Respir Crit Care Med 2002; 166: S25-S30.
37 Baraldi E, Bonetto G, Zacchello F, et al. Low exhaled nitric oxide in school-age children with bronchopulmonary dysplasia and airflow limitation. Am J Respir Crit Care Med 2005; 171: 68-72.

38 Dweik RA, Boggs PB, Erzurum SC, et al. An official ATS clinical practice guideline: interpretation of exhaled nitric oxide levels (FeNO) for clinical applications. Am J Respir Crit Care Med 2011; 184: 602-615.

39 Agusti A, MacNee W, Donaldson K, et al. Hypothesis: does COPD have an autoimmune component? Thorax 2003; 58: 832-834.

40 Cosio MG, Saetta M, Agusti A. Immunologic aspects of chronic obstructive pulmonary disease. N Engl J Med 2009; 360: 2445-2454. 